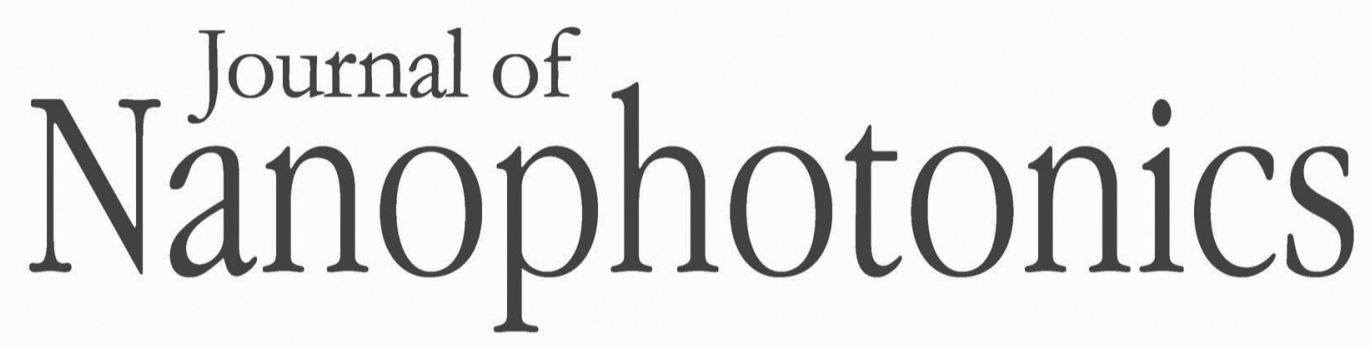

\title{
Characteristics of carrier injection on polarization-dependent photoexcitation in copper phthalocyanine thin films
}

Hyung Keun Yoo

Chul-Sik Kee

Chul Kang

In-Wook Hwang

Joong Wook Lee

\section{O SPIE}




\title{
Characteristics of carrier injection on polarization-dependent photoexcitation in copper phthalocyanine thin films
}

\author{
Hyung Keun Yoo, ${ }^{\text {a }}$ Chul-Sik Kee, ${ }^{a}$ Chul Kang, ${ }^{a}$ In-Wook Hwang, ${ }^{a}$ and \\ Joong Wook Lee ${ }^{\text {b }}$ \\ ${ }^{\mathrm{a}}$ Gwangju Institute of Science and Technology, Advanced Photonics Research Institute, \\ Gwangju 500-712, Republic of Korea \\ ${ }^{\mathrm{b}}$ Chonnam National University, Department of Physics, Gwangju 500-757, Republic of Korea \\ leejujc@chonnam.ac.kr
}

\begin{abstract}
The carrier concentration injected from a silicon substrate to a copper phthalocyanine thin film was found to depend on the incidence polarization of the photoexciting beam. The modulation efficiency of terahertz transmission due to transverse-magnetic (TM)-polarized excitation is distinctly higher than that due to transverse-electric (TE)-polarized excitation. Underlying this difference is the enhancement of carrier injection when the TM-polarized light is more transmitted through the surface of organic thin films than the TE-polarization light. () The Authors. Published by SPIE under a Creative Commons Attribution 3.0 Unported License. Distribution or reproduction of this work in whole or in part requires full attribution of the original publication, including its DOI. [DOI: 10.1117/1.JNP.7.073795]
\end{abstract}

Keywords: terahertz spectroscopy; terahertz modulation; organic thin film; polarizationdependent transmission.

Paper 13041SSP received Jul. 26, 2013; revised manuscript received Aug. 22, 2013; accepted for publication Sep. 17, 2013; published online Oct. 8, 2013.

\section{Introduction}

Terahertz (THz) technologies for $\mathrm{THz}$ devices and $\mathrm{THz}$ imaging systems have developed considerably. ${ }^{1}$ Many researchers have extensively investigated for developing $\mathrm{THz}$ devices such as $\mathrm{THz}$ filters, switches, mirrors, and modulators. ${ }^{2-9}$ In particular, active $\mathrm{THz}$ modulation over a broad $\mathrm{THz}$ range became an important function for versatile $\mathrm{THz}$ devices. ${ }^{8-12}$ For active $\mathrm{THz}$ modulation, quantum-well or plasmonic structures were employed, ${ }^{3,7}$ and metamaterials based on hybrid structures of metals and semiconductors were also used to obtain a relatively high modulation efficiency. ${ }^{5,13,14}$ Very recently, a new method for active $\mathrm{THz}$ modulation has been reported by Yoo et al., who demonstrated highly efficient $\mathrm{THz}$ modulations over a wide spectral range by using organic copper phthalocyanine $(\mathrm{CuPc})$ thin films deposited on a silicon ( $\mathrm{Si}$ ) substrate. ${ }^{15,16}$ The novelty of this paper is to understand the characteristics of $\mathrm{THz}$ modulation efficiency under the condition of different polarization of the photoexciting beam, which is important for wide applications of the new method for active $\mathrm{THz}$ wave modulation.

In this article, we present the characteristics of $\mathrm{THz}$ modulation on transmission through organic-inorganic hybrid structures which are composed of a 500-nm-thick CuPc thin film and a $500-\mu \mathrm{m}$-thick $\mathrm{Si}$ substrate. We report that the $\mathrm{THz}$ modulation efficiency is related to the carrier concentration injected from the Si substrate to the CuPc thin film, which depends on the incidence polarization of the photoexciting beam. The modulation efficiency of THz transmission is relatively high when the photocarriers are excited by a transverse-magnetic (TM)-polarized incident light, compared with the case of TE-polarized incident light. This phenomenon is explained by the enhancement of carrier injection which is expected since the TM-polarized light is more transmitted through the surface of organic thin films than the transverse-electric (TE)-polarization light. 
(a)

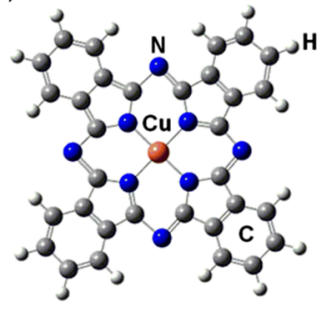

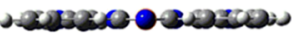

(c)

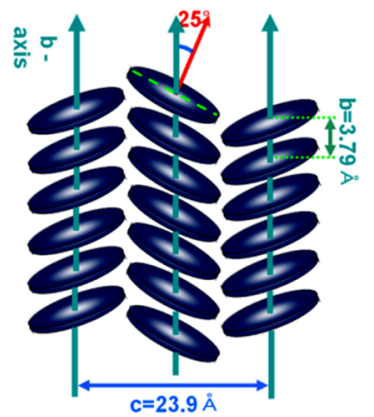

(b)

CuPc stacking at Room Temp.

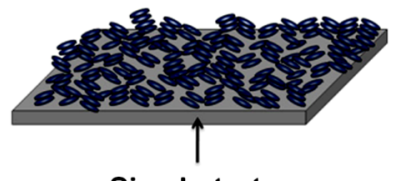

Si substrate

(d)

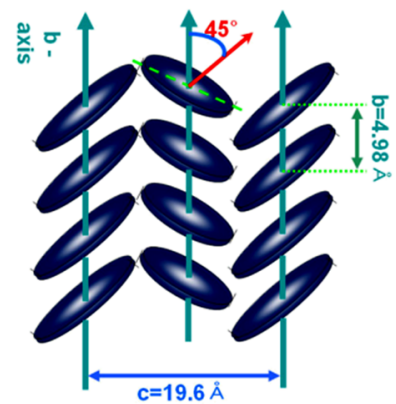

Fig. 1 (a) Schematic diagram of the molecular structure of CuPc. (b) Stacking structure of CuPc molecules, deposited on intrinsic silicon substrate, annealed at $27^{\circ} \mathrm{C}$. (c) and (d) The crystalline $\alpha$ - and $\beta$-phases of CuPc molecules, respectively.

\section{Basic Properties and Fabrication of CuPc Thin Films}

In this study, the $\mathrm{CuPc}$ powder $\beta$-form (Sigma-Aldrich) was used to fabricate CuPc films. Figure 1(a) shows the chemical structure of a CuPc molecule which consists of a core metal ion and a chelate organic ring with four nitrogen atoms. CuPc films of 500-nm thickness were deposited on a Si substrate with thickness of $500 \mu \mathrm{m}$, using thermal evaporation method. Three different types of samples were prepared at the annealing temperature, $27^{\circ} \mathrm{C}$ (room temperature), $150^{\circ} \mathrm{C}$, and $250^{\circ} \mathrm{C}$, respectively. The sample fabricated at $27^{\circ} \mathrm{C}$ shows randomly stacked CuPc molecules, as depicted in Fig. 1(b). As the annealing temperature increases, the column-like structures formed by stacking $\mathrm{CuPc}$ molecules together are increasingly crystallized, as shown in Figs. 1(c) and 1(d). The well-ordered $\alpha$ - and $\beta$-phase CuPc films are fabricated under the conditions of temperatures of $150^{\circ} \mathrm{C}$ and $250^{\circ} \mathrm{C}$, respectively. ${ }^{17-19} \mathrm{~A}$ main difference of two phases is the angle between b-axes and orthogonal axes to molecular planes, ${ }^{20}$ which of necessity causes the change of the direction of molecular stacking and its stacking density. Moreover, the structural change may be associated with the characteristics of carrier injection between the $\mathrm{CuPc}$ thin films and the $\mathrm{Si}$ substrate and charge transport in the CuPc films, thus altering the absorption properties of the incident electromagnetic waves. ${ }^{21-26}$

\section{THz Time-Domain Spectroscopy and Transmission Measurements}

To measure the characteristics of $\mathrm{THz}$ transmission through the hybrid structures of the CuPc thin films and the $\mathrm{Si}$ substrate, we used a standard THz time-domain spectroscopy system. ${ }^{27,28}$ $\mathrm{THz}$ pulses are generated by using a p-type InAs wafer with (100) orientation and detected by using a photoconductive antenna method. The generated $\mathrm{THz}$ pulses are collimated by using two parabolic mirrors. The $\mathrm{THz}$ pulses are normally incident to the $\mathrm{CuPc} / \mathrm{Si}$ hybrid structures, and a continuous optical beam is obliquely incident with the incidence angle of $75 \mathrm{deg}$ at the same time. We used the laser beam with the wavelength of $785 \mathrm{~nm}$ at which the carriers are strongly excited on the Si substrate and the absorption on the CuPc thin film can be strongly suppressed. ${ }^{29}$ 


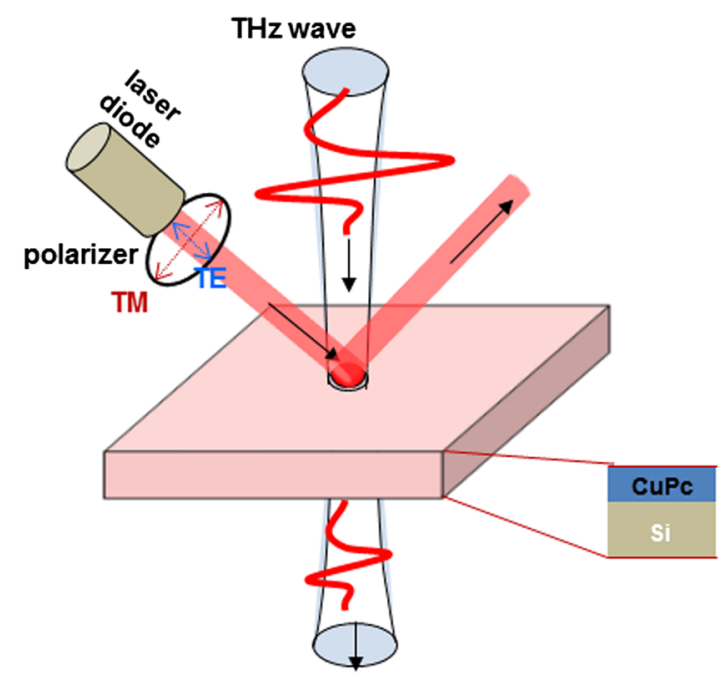

Fig. 2 Schematic view of an experiment of the $\mathrm{THz}$ wave transmission through a CuPc film on $\mathrm{Si}$ under optical excitation. The optical beam for photoexcitation is obliquely incident with the incidence angle of $75 \mathrm{deg}$, and the $\mathrm{THz}$ pulses are normally incident to the surface of the samples.

The polarization of the incident optical beam is changed to be TM- and TE-polarized light, as shown in Fig. 2, by rotating a polarizer. In the case of the TM-polarized light, the electric fields are parallel to an incidence plane, whereas in the case of the TE-polarized light, the electric fields are normal to the incidence plane.

\section{Polarization-Dependent Transmission Modulation of THz Waves in CuPc Thin Films}

Figure 3 shows the peak values of normalized $\mathrm{THz}$ transmission of four different samples, a bare $\mathrm{Si}$ substrate and $\mathrm{CuPc} / \mathrm{Si}$ hybrid structures prepared at different temperatures, $27^{\circ} \mathrm{C}$ (room temperature), $150^{\circ} \mathrm{C}$, and $250^{\circ} \mathrm{C}$, on the incident polarization and power of the optical beams for photoexcitation. The peak values of transmission amplitudes measured under the condition of

TM Polarization

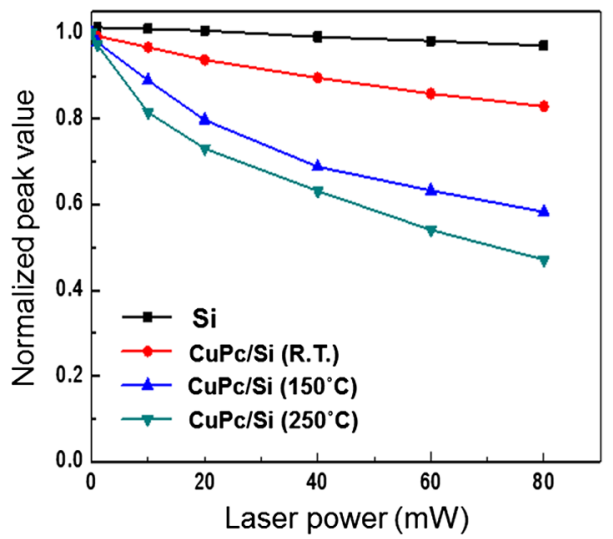

TE Polarization

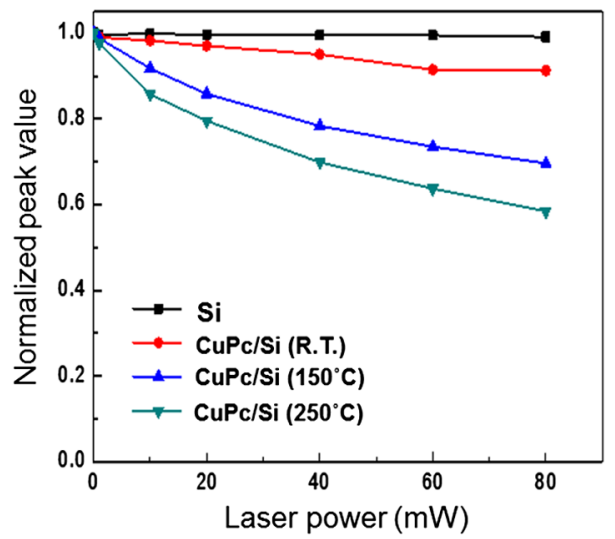

Fig. 3 (a) Peak values of normalized THz transmission through the samples, a Si substrate (black squares) and hybrid $\mathrm{CuPc} / \mathrm{Si}$ layers annealed at $27^{\circ} \mathrm{C}$ (red circles), $150^{\circ} \mathrm{C}$ (blue triangles), and $250^{\circ} \mathrm{C}$ (green inverted triangles), respectively, measured under the condition of TM-polarized excitation of incident optical beam. (b) Peak values of normalized $\mathrm{THz}$ transmission measured under the condition of TE-polarized excitation of incident optical beam. The laser power for photoexcitation is varied from zero to $80 \mathrm{~mW}$. 


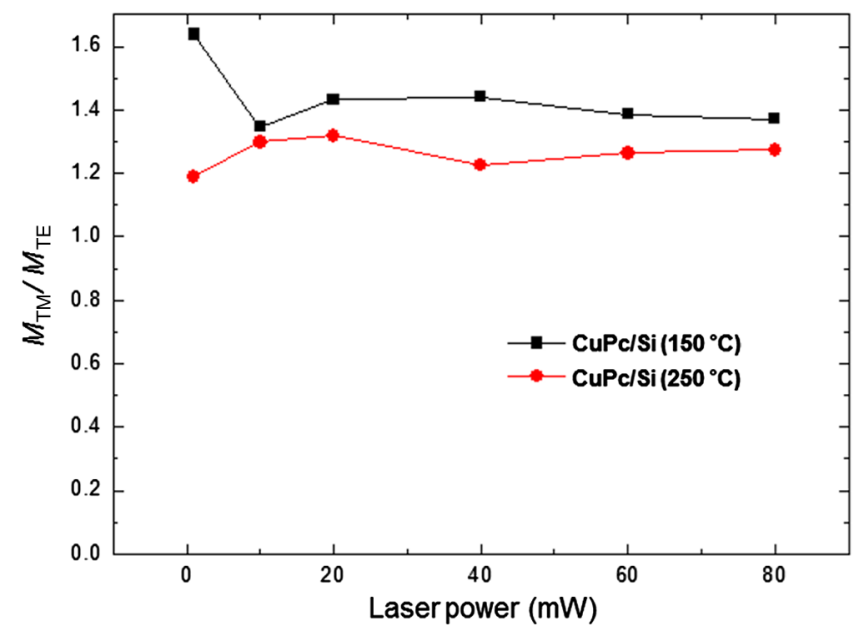

Fig. 4 The ratio between $M_{\mathrm{TM}}$ (the modulation efficiency due to TM photoexcitation) and $M_{\mathrm{TE}}$ (the modulation efficiency due to TE photoexcitation) for $\alpha$ - (black squares) and $\beta$ - (red circles) phase CuPc films.

photoexcitation were normalized by ones measured without photoexcitation. As we easily expect, the transmission peaks may decrease with increasing the power of the optical beams as well as with improving the ordering properties of the crystallizing $\mathrm{CuPc}$ molecules. ${ }^{15} \mathrm{At}$ the photoexciting power of $80 \mathrm{~mW}$ and the well-ordered structures of CuPc molecules, the peak value is $<50 \%$. Note that the peak values observed under the condition of TM-polarized excitation are relatively higher than those obtained under the condition of TE-polarized excitation.

To quantitatively compare the effects of differently polarized waves on $\mathrm{THz}$ modulation, we used the term modulation efficiency, $M=\left(P_{w / o}-P_{w}\right) / P_{w / o}$, where $P_{w / o}$ and $P_{w}$ are the peak values of transmission amplitudes measured without and with photoexcitation, respectively. ${ }^{15}$ Figure 4 shows the ratio between the modulation efficiencies, $M_{\mathrm{TE}}$ and $M_{\mathrm{TM}}$, in cases of TEand TM-polarized optical beams. Only the values obtained at well-ordered $\alpha$ - and $\beta$-phase $\mathrm{CuPc}$ films with relatively high modulation efficiencies are compared. At higher laser powers, the ratio can be reliably extracted since the values of modulation efficiency are relatively high. On the other hand, at lower laser powers, both the values of modulation efficiency in TMand TE-polarized cases are too small. The ratio between two small numbers is therefore likely to be inaccurate. Nevertheless, the results at lower laser powers are quite consistent with those at higher laser powers. The modulation efficiencies for the TM polarization are $>30 \%$ as large as those for the TE polarization. The reflectivity in the case when the electric field is perpendicular to the plane of incidence is larger than that in the case when the electric field is parallel to the plane of incidence on the surface of a CuPc thin film. This means that much more power of the incident optical beam for photoexcitation reaches the Si substrate and therefore the concentration of the photoexcited carriers will be increased. The enhancement of carrier injection from a Si substrate to a CuPc thin film and the amount of carrier transport within the CuPc film will be followed. The density of photoexcited carriers may increase and the incident $\mathrm{THz}$ waves are strongly modulated consequently. This may improve the modulation efficiency of $\mathrm{THz}$ transmission.

\section{Conclusions}

In conclusion, we have demonstrated that the carrier concentration injected from a silicon substrate to a $\mathrm{CuPc}$ thin film depends on the incidence polarization of the photoexciting beam. The modulation efficiency of $\mathrm{THz}$ transmission due to TM-polarized excitation is distinctly higher than that due to TE-polarized excitation. We have found that this phenomenon is due to the increase of the concentration of the photoexcited carriers, which is expected when the incident optical beams are more transmitted through the organic thin film. 


\section{Acknowledgments}

This research was supported by Basic Science Research Program through the National Research Foundation of Korea (NRF) funded by the Ministry of Education, Science and Technology (2010-0021181; NRF-2012R1A6A3A01018114). This study was financially supported by Chonnam National University in 2013 and Research Program (Applications of Ultrashort Quantum Beam Facility) through a grant provided by the GIST in 2013. Hyung Keun Yoo made the samples and obtained the transmission signals, Chul Kang helped to obtain the transmission signals from THz time-domain spectroscopy system, Chul-Sik Kee and In-Wook Hwang analyzed the signals, and Joong Wook Lee directed this study, analyzed the results, and wrote this manuscript.

\section{References}

1. M. Tonouchi, "Cutting-edge terahertz technology," Nat. Photonics 1(2), 97-105 (2007), http://dx.doi.org/10.1038/nphoton.2007.3.

2. J. N. Heyman, R. Kersting, and K. Unterrainer, "Time-domain measurement of intersubband oscillations in a quantum well," Appl. Phys. Lett. 72(6), 644-646 (1998), http://dx.doi .org/10.1063/1.120832.

3. I. H. Libon et al., "An optically controllable terahertz filter," Appl. Phys. Lett. 76(20), 28212823 (2000), http://dx.doi.org/10.1063/1.126484.

4. T. Kleine-Ostmann et al., "Room-temperature operation of an electrically driven terahertz modulator," Appl. Phys. Lett. 84(18), 3555-3557 (2004), http://dx.doi.org/10.1063/1 .1723689 .

5. H.-T. Chen et al., "Active terahertz metamaterial devices," Nature 444, 597-600 (2006), http://dx.doi.org/10.1038/nature05343.

6. E. Hendry et al., "Ultrafast optical switching of the $\mathrm{THz}$ transmission through metallic subwavelength hole arrays," Phys. Rev. B 75, 235305 (2007), http://dx.doi.org/10.1103/ PhysRevB.75.235305.

7. E. Hendry et al., "Optical control over surface-plasmon-polariton-assisted THz transmission through a slit aperture," Phys. Rev. Lett. 100(12), 123901 (2008), http://dx.doi.org/10.1103/ PhysRevLett.100.123901.

8. H.-T. Chen et al., "A metamaterial solid-state terahertz phase modulator," Nat. Photonics 3(3), 148-151 (2009), http://dx.doi.org/10.1038/nphoton.2009.3.

9. D. G. Cooke and P. Uhd Jepsen, "Optical modulation of terahertz pulses in a parallel plate waveguide,” Opt. Express 16(19), 15123-15129 (2008), http://dx.doi.org/10.1364/OE.16 .015123 .

10. T. Kleine-Ostmann et al., "Audio signal transmission over $\mathrm{THz}$ communication channel using semiconductor modulator," Electron. Lett. 40(2), 124-126 (2004), http://dx.doi .org/10.1049/el:20040106.

11. W. L. Chan et al., "A spatial light modulator for terahertz beams," Appl. Phys. Lett. 94(21), 213511 (2009), http://dx.doi.org/10.1063/1.3147221.

12. S. Zhang et al., "Photoinduced handedness switching in terahertz chiral metamolecules," Nat. Commun. 3, 942 (2012), http://dx.doi.org/10.1038/ncomms1908.

13. M. A. Seo et al., "Active terahertz nanoantennas based on VO2 phase transition," Nano Lett. 10(6), 2064-2068 (2010), http://dx.doi.org/10.1021/nl1002153.

14. S. H. Lee et al., "Switching terahertz waves with gate-controlled active graphene metamaterials," Nat. Mater. 11, 936-941 (2012), http://dx.doi.org/10.1038/nmat3433.

15. H. K. Yoo et al., "Organic conjugated material-based broadband terahertz wave modulators," Appl. Phys. Lett. 99(6), 061108 (2011), http://dx.doi.org/10.1063/1.3626591.

16. H. K. Yoo et al., "Transmittances of terahertz pulses through organic copper phthalocyanine films on Si under optical carrier-excitation," Appl. Phys. Express 5(7), 072402 (2012), http://dx.doi.org/10.1143/APEX.5.072402.

17. G. Guillaud, J. Simon, and J. P. Germain, "Metallophthalocyanines: gas sensors, resistors and field effect transistors," Coordin. Chem. Rev. 178(Part 2), 1433-1484 (1998), http://dx .doi.org/10.1016/S0010-8545(98)00177-5. 
18. M. J. Cook and I. Chambrier, The Porphyrin Handbook, Elsevier Science, New York (2003).

19. F. Iwatsu, "Size effects on the alpha-beta transformation of phthalocyanine crystals," J. Phys. Chem. 92(6), 1678-1681 (1988), http://dx.doi.org/10.1021/j100317a057.

20. R. Mason, G. A. Williams, and P. E. Fielding, "Structural chemistry of phthalocyaninato cobalt (II) and manganese (II)," J. Chem. Soc. Dolton Trans. 4, 676-683 (1979), http://dx .doi.org/10.1039/dt9790000676.

21. S. Karan and B. Mallik, "Nanostructured organic-inorganic photodiodes with high rectification ratio," Nanotechnology 19(49), 495202 (2008), http://dx.doi.org/10.1088/09574484/19/49/495202.

22. R. D. Gould and A. K. Hassan, "AC electrical properties of thermally evaporated thin films of copper phthalocyanine," Thin Solid Films 223(2), 334-340 (1993), http://dx.doi.org/10 .1016/0040-6090(93)90541-V.

23. P. Peumans, S. Uchida, and S. R. Forrest, "Efficient bulk heterojunction photovoltaic cells using small-molecular-weight organic thin films," Nature 425(6954), 158-162 (2003), http://dx.doi.org/10.1038/nature01949.

24. H. Fujikake et al., "Time-of-flight analysis of charge mobility in a $\mathrm{Cu}$-phthalocyanine-based discotic liquid crystal semiconductor,' Appl. Phys. Lett. 85(16), 3474-3476 (2004), http:// dx.doi.org/10.1063/1.1805178.

25. S. Ambily and C. S. Menon, "The effect of growth parameters on the electrical, optical and structural properties of copper phthalocyanine thin films," Thin Solid Films 347(1), 284-288 (1999), http://dx.doi.org/10.1016/S0040-6090(98)01744-1.

26. M. D. Pirriera et al., "Optoelectronic properties of $\mathrm{CuPc}$ thin films deposited at different substrate temperatures," J. Phys. D: Appl. Phys. 42(14), 145102 (2009), http://dx.doi.org/10 $.1088 / 0022-3727 / 42 / 14 / 145102$.

27. M. van Exter and D. Grischkowsky, "Optical and electronic properties of doped silicon from 0.1 to 2 THz," Appl. Phys. Lett. 56(17), 1694-1696 (1990), http://dx.doi.org/10.1063/1 .103120 .

28. Z. Jiang, M. Li, and X. C. Zhang, "Dielectric constant measurement of thin films by differential time-domain spectroscopy," Appl. Phys. Lett. 76(22), 3221-3223 (2000), http://dx.doi .org/10.1063/1.126587.

29. Y. Nakato, M. Shioji, and H. Tsubomura, "Photovoltage and stability of an n-type silicon semiconductor coated with metal or metal-free phthalocyanine thin films in aqueous redox solutions," J. Phys. Chem. 85(12), 1670-1672 (1981), http://dx.doi.org/10.1021/ j150612a014.

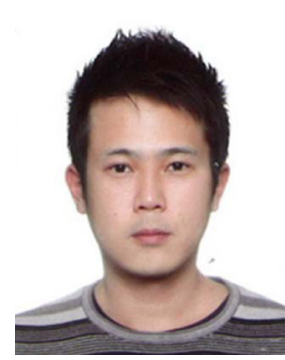

Hyung Keun Yoo is a postdoctoral researcher of the Advanced Photonics Research Institute at Gwangju Institute of Science and Technology (GIST), Gwangju, Republic of Korea. He has investigated terahertz waves spectroscopy and application based on carrier dynamics in optically excited states of organic semiconductors. He graduated from Sogang University with a BS in 2004 and an MS in 2006 in physics and received a PhD from Sogang University in 2012.

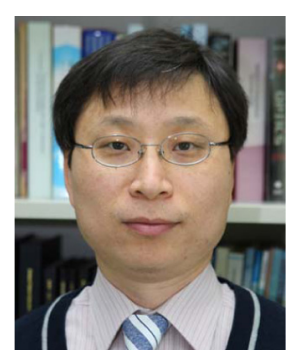

Chul-Sik Kee is a principal researcher of the Advanced Photonics Research Institute (APRI) at Gwangju Institute of Science and Technology (GIST), Republic of Korea. He received his PhD degree in physics at Korea Advanced Institute of Science and Technology (KAIST), Korea (2000). He worked as a postdoctoral fellow in the Department of Electrical Engineering at the University of California Los Angeles (UCLA), USA (2000 to 2001), a research professor in Ajou University, Korea (2001 to 2003), and a senior researcher in Electronics and Telecommunications Research Institute (ETRI), Republic of Korea (2003 to 2004). He has been with the Nanophotonics Laboratory of APRI since 2004. 
His research has included photonic crystals, metamaterials, and terahertz pulse generation from nanostructures.

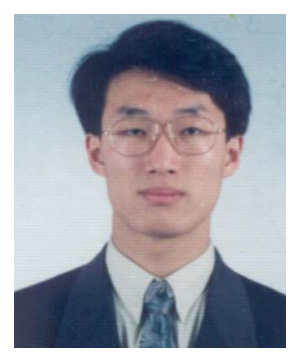

Chul Kang is a senior research scientist of Advanced Photonics Research Institute at Gwangju Institute of Science and Technology (GIST), Gwangju, Republic of Korea, where he develops terahertz generation and spectroscopy for semiconductors and nanomaterials. He received his BS degree in physics in 2001 and his $\mathrm{PhD}$ degree in physics in 2006 at University of Seoul.

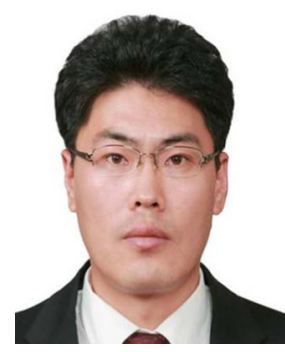

In-Wook Hwang is a senior research scientist of Advanced Photonics Research Institute at Gwangju Institute of Science and Technology (GIST), Republic of Korea. His research fields are ultrafast laser spectroscopy and organic solar cells. He received his $\mathrm{PhD}$ degree in chemistry at Yonsei University 2002. In the period of 2002 to 2009, he worked as a researcher in Yonsei University, UCSB, and GIST, characterizing ultrafast energy and electron dynamics in various organic systems (porphyrin arrays, liquid crystals, and semiconducting polymers) and fabricating high-performance solar cells.

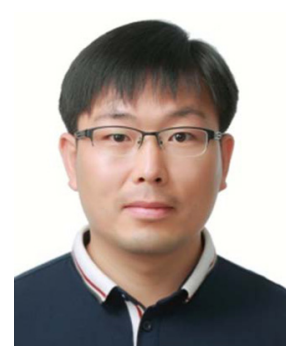

Joong Wook Lee is an assistant professor in Department of Physics at Chonnam National University (CNU), Gwangju, Korea, where he develops terahertz sensing \& imaging systems for biological applications and terahertz devices for communications. He received his BS degree in earth \& environments science in 2001 and his $\mathrm{PhD}$ degree in physics in 2006 at Seoul National University. He worked as a postdoc in Department of Chemistry at Seoul National University, developing nanoparticle-based biosensors and supported lipid bilayer systems, for the following oneyear period. After that, he worked as a postdoc at Electrical \& Computer Engineering, Rice University, Houston, Texas, to 2010 and as a senior research scientist of Advanced Photonics Research Institute at Gwangju Institute of Science and Technology (GIST) to 2013. 